\title{
On the relative fundamental solutions for a second order differential operator on the Heisenberg group
}

by

\author{
T. Godoy and L. SAAL (Cordoba)
}

\begin{abstract}
Let $H_{n}$ be the $(2 n+1)$-dimensional Heisenberg group, let $p, q \geq 1$ be integers satisfying $p+q=n$, and let

$$
L=\sum_{j=1}^{p}\left(X_{j}^{2}+Y_{j}^{2}\right)-\sum_{j=p+1}^{n}\left(X_{j}^{2}+Y_{j}^{2}\right),
$$

where $\left\{X_{1}, Y_{1}, \ldots, X_{n}, Y_{n}, T\right\}$ denotes the standard basis of the Lie algebra of $H_{n}$. We compute explicitly a relative fundamental solution for $L$.
\end{abstract}

1. Introduction. Let $n \geq 2$ and let $p, q \geq 1$ be a pair of integers such that $p+q=n$. Let $H_{n}$ be the Heisenberg group defined by $H_{n}=\mathbb{C}^{n} \times \mathbb{R}$ with group law $(z, t)\left(z^{\prime}, t^{\prime}\right)=\left(z+z^{\prime}, t+t^{\prime}-\frac{1}{2} \operatorname{Im} B\left(z, z^{\prime}\right)\right)$ where $B(z, w)=$ $\sum_{j=1}^{p} z_{j} \bar{w}_{j}-\sum_{j=p+1}^{n} z_{j} \bar{w}_{j}$. For $x=\left(x_{1}, \ldots, x_{n}\right) \in \mathbb{R}^{n}$, we write $x=\left(x^{\prime}, x^{\prime \prime}\right)$ with $x^{\prime} \in \mathbb{R}^{p}, x^{\prime \prime} \in \mathbb{R}^{q}$. So, $\mathbb{R}^{2 n}$ can be identified with $\mathbb{C}^{n}$ via the map $\varphi\left(x^{\prime}, x^{\prime \prime}, y^{\prime}, y^{\prime \prime}\right)=\left(x^{\prime}+i y^{\prime}, x^{\prime \prime}-i y^{\prime \prime}\right), x^{\prime}, y^{\prime} \in \mathbb{R}^{p}, x^{\prime \prime}, y^{\prime \prime} \in \mathbb{R}^{q}$. In this setting the form $-\operatorname{Im} B(z, w)$ agrees with the standard symplectic form on $\mathbb{R}^{2(p+q)}$, and the vector fields

$$
X_{j}=-\frac{1}{2} y_{j} \frac{\partial}{\partial t}+\frac{\partial}{\partial x_{j}}, \quad Y_{j}=\frac{1}{2} x_{j} \frac{\partial}{\partial t}+\frac{\partial}{\partial y_{j}}, \quad j=1, \ldots, n,
$$

and $T=\partial / \partial t$ form the standard basis for the Lie algebra $h_{n}$ of $H_{n}$. Thus $H_{n}$ can be viewed as $\mathbb{R}^{n} \times \mathbb{R}^{n} \times \mathbb{R}$ via the map $(x, y, t) \mapsto(\varphi(x, y), t)$. From now on, we will use freely this identification.

As usual we denote by $S\left(H_{n}\right)$ the Schwartz space on $H_{n}$ and by $S^{\prime}\left(H_{n}\right)$ the space of corresponding tempered distributions.

Let $L=\sum_{j=1}^{p}\left(X_{j}^{2}+Y_{j}^{2}\right)-\sum_{j=p+1}^{n}\left(X_{j}^{2}+Y_{j}^{2}\right)$, so $L$ has a self-adjoint extension (that we still denote by $L$ ), as an operator densely defined on $L^{2}\left(H_{n}\right)$. Let $K$ be the (closed) kernel of $L$ in $L^{2}\left(H_{n}\right)$ and let $\pi$ be the

2000 Mathematics Subject Classification: Primary 43A80; Secondary 35A08.

Research partially supported by CONICOR, SECYT UNC and CONICET. 
orthogonal projection on $K$. We recall that $\Phi \in S^{\prime}\left(H_{n}\right)$ is a relative fundamental solution for $L$ if

$$
L(f * \Phi)=L(f) * \Phi=f-\pi(f) \quad \text { for all } f \in S\left(H_{n}\right) .
$$

In $[M-R 1]$ and $[M-R 2]$ it is proved that there exist relative fundamental solutions for a wide class of left invariant second order differential operators that includes $L$. On the other hand, for the case $q=0$ (i.e. the case $L=$ $\left.\sum_{j=1}^{n}\left(X_{j}^{2}+Y_{j}^{2}\right)\right)$, a fundamental solution is computed in [F1].

Consider the natural action of $U(p, q)$ on $H_{n}$ given by $g \cdot(z, t)=(g z, t)$. So $U(p, q)$ acts on $L^{2}\left(H_{n}\right), S\left(H_{n}\right)$ and $S^{\prime}\left(H_{n}\right)$ in the canonical way.

In [G-S] it is proved that there exists a family of tempered $U(p, q)$ invariant distributions $S_{\lambda, k}, \lambda \in \mathbb{R}-\{0\}, k \in \mathbb{Z}$, satisfying

$$
L S_{\lambda, k}=-|\lambda|(2 k+p-q) S_{\lambda, k}, \quad i T S_{\lambda, k}=\lambda S_{\lambda, k} .
$$

It is also proved that the solution space in $S^{\prime}\left(H_{n}\right)^{U(p, q)}$ of the system (1.1) is one-dimensional (see also [F2] and [H-T]) and that the distributions $S_{\lambda, k}$ defined there satisfy

$$
f=\sum_{k \in \mathbb{Z}} \int_{-\infty}^{\infty} f * S_{\lambda, k}|\lambda|^{n} d \lambda
$$

for all $f \in S\left(H_{n}\right)$. The convolution product is given, as usual, by the formula $\left(f * S_{\lambda, k}\right)(z, t)=\left\langle S_{\lambda, k},\left(L_{(z, t)^{-1}} f\right)^{\vee}\right\rangle$, where we write, for $g: H_{n} \rightarrow \mathbb{C}$,

$$
\left(L_{(z, t)^{-1}} g\right)\left(z^{\prime}, t^{\prime}\right)=g\left((z, t)^{-1}\left(z^{\prime}, t^{\prime}\right)\right) \quad \text { and } \quad g^{\vee}((z, t))=g\left((z, t)^{-1}\right) .
$$

In this setting, it is not hard to show (see Lemma 2.1 below) that $\Phi$ defined by

$$
\langle\Phi, f\rangle=\sum_{2 k+p-q \neq 0} \frac{-1}{2 k+p-q} \int_{-\infty}^{\infty}\left\langle S_{\lambda, k}, f\right\rangle|\lambda|^{n-1} d \lambda
$$

is a well defined element in $S^{\prime}\left(H_{n}\right)$ and that $\Phi$ is a relative fundamental solution for $L$.

Let us introduce some notation and recall some known facts. Let $\mathcal{H}$ be the space of functions $\varphi: \mathbb{R} \rightarrow \mathbb{C}$ such that $\varphi(\tau)=\varphi_{1}(\tau)+H(\tau) \varphi_{2}(\tau) \tau^{n-1}$, where $\varphi_{1}, \varphi_{2} \in S(\mathbb{R})$ and $H$ denotes the Heaviside function, i.e. $H(\tau)=$ $\chi_{(0, \infty)}(\tau)$. It is proved in $[\mathrm{T}]$ that $\mathcal{H}$ provided with a suitable topology is a Fréchet space. Also, for $p+q=n, p, q \geq 1$, there is given a continuous linear surjective map $N: S\left(\mathbb{R}^{n}\right) \rightarrow \mathcal{H}$ such that its adjoint $N^{\prime}: \mathcal{H}^{\prime} \rightarrow S^{\prime}\left(\mathbb{R}^{n}\right)^{O(p, q)}$ is a linear homeomorphism onto the space of $O(p, q)$-invariant tempered distributions on $\mathbb{R}^{n}$. As pointed out in [G-S], this construction also works for the space $S^{\prime}\left(\mathbb{C}^{n}\right)^{U(p, q)}$, i.e., there exists a continuous linear surjective map $N: S\left(\mathbb{C}^{n}\right) \rightarrow \mathcal{H}$ whose adjoint $N^{\prime}: \mathcal{H}^{\prime} \rightarrow S^{\prime}\left(\mathbb{C}^{n}\right)^{U(p, q)}$ is a homeomorphism. 
From now on, $N: S^{\prime}\left(\mathbb{C}^{n}\right) \rightarrow S^{\prime}(\mathbb{R})$ will be the operator given by (2.11) of [G-S]. For $f \in S\left(H_{n}\right)$, we write $N f(\tau, t)$ for $N(f(\cdot, t))(\tau)$.

Our aim in this paper is to obtain a rather explicit description of the relative fundamental solution $\Phi$ for $L$. In Theorem 4.10 we will compute a distribution $\mu \in S^{\prime}\left(\mathbb{R}^{2}\right)$ satisfying $\langle\Phi, f\rangle=\langle\mu, N f\rangle, f \in S\left(H_{n}\right)$.

Acknowledgments. We express our thanks to Fulvio Ricci, who inspired this work and kindly helped us during its preparation.

2. Notations and preliminaries. For $\lambda \in \mathbb{R}-\{0\}$, let $\pi_{\lambda}$ be the Schrödinger representation on $L^{2}\left(\mathbb{R}^{n}\right)$ of the Heisenberg group $\mathbb{R}^{n} \times \mathbb{R}^{n} \times \mathbb{R}$ defined by

$$
\pi_{\lambda}(x, y, t) h(\zeta)=e^{-i\left(\lambda t+\operatorname{sgn}(\lambda) \sqrt{|\lambda|} x \cdot \zeta+\frac{1}{2} \lambda x \cdot y\right)} h(\zeta+\sqrt{|\lambda|} y) .
$$

We still denote by $\pi_{\lambda}$ the corresponding representation of $H_{n}=\mathbb{C}^{n} \times \mathbb{R}$ (via the map $\varphi$ of the introduction). For $f \in S\left(H_{n}\right)$, we set

$$
\pi_{\lambda}(f)=\int_{H_{n}} f(z, t) \pi_{\lambda}(z, t)^{-1} d z d t
$$

where $d z d t$ means the Lebesgue measure on $\mathbb{C}^{n} \times \mathbb{R}$. For $f \in S\left(H_{n}\right)$ and $h_{1}, h_{2} \in L^{2}\left(\mathbb{R}^{n}\right)$, let $E_{\lambda}\left(h_{1}, h_{2}\right)$ be the associated matrix entry given by

$$
E_{\lambda}\left(h_{1}, h_{2}\right)(z, t)=\left\langle\pi_{\lambda}(z, t) h_{1}, h_{2}\right\rangle .
$$

For $\alpha=\left(\alpha_{1}, \ldots, \alpha_{n}\right) \in(\mathbb{N} \cup\{0\})^{n}$, let $h_{\alpha}$ be the Hermite function given by

$$
h_{\alpha}(\zeta)=\left(2^{|\alpha|} \alpha ! \sqrt{\pi}\right)^{-n / 2} e^{-|\zeta|^{2} / 2} \prod_{j=1}^{n} H_{\alpha_{j}}\left(\zeta_{j}\right)
$$

with $|\alpha|=\alpha_{1}+\ldots+\alpha_{n}, \alpha !=\alpha_{1} ! \ldots \alpha_{n}$ ! and where

$$
H_{k}(s)=(-1)^{k} e^{s^{2}} \frac{d^{k}}{d s^{k}}\left(e^{-s^{2}}\right)
$$

is the $k$ th Hermite polynomial. Finally we also set

$$
\|\alpha\|=\sum_{j=1}^{p} \alpha_{j}-\sum_{j=p+1}^{n} \alpha_{j} .
$$

We also recall that, for nonnegative integers $m, k$ with $k \leq m$, the Laguerre polynomials $L_{m}^{k}(x)$ are defined by (see e.g. [Sz])

$$
L_{m}^{0}(t)=\sum_{j=0}^{m}\left(\begin{array}{c}
m \\
j
\end{array}\right)(-1)^{j} \frac{t^{j}}{j !}, \quad L_{m-1}^{\beta+1}(t)=-\frac{d}{d t} L_{m}^{\beta}(t) .
$$

The distributions $S_{\lambda, k}$ are defined in [G-S] by

$$
\left\langle S_{\lambda, k}, f\right\rangle=\sum_{\|\alpha\|=k}\left\langle E_{\lambda}\left(h_{\alpha}, h_{\alpha}\right), f\right\rangle .
$$


It is proved (see [G-S], Theorem 4.1 and Remarks $4.2,4.3$ ) that $S_{\lambda, k}=$ $F_{\lambda, k} \otimes e^{-i \lambda t}$, where $F_{\lambda, k} \in S^{\prime}\left(\mathbb{C}^{n}\right)$ is described, in terms of the Laguerre polynomials and the operator $N$, by

$$
\begin{aligned}
& \left\langle F_{\lambda, k}, g\right\rangle \\
& \quad=\left\langle\left(L_{k-q+n-1}^{0} H\right)^{(n-1)}, \tau \mapsto \frac{2}{|\lambda|} e^{-\tau / 2} N g\left(\frac{2}{|\lambda|} \tau\right)\right\rangle, \quad g \in S\left(\mathbb{C}^{n}\right),
\end{aligned}
$$

for $k \geq q, \lambda \neq 0$,

$$
\begin{aligned}
& \left\langle F_{\lambda, k}, g\right\rangle \\
= & \left\langle\left(L_{-k-p+n-1}^{0} H\right)^{(n-1)}, \tau \mapsto \frac{2}{|\lambda|} e^{-\tau / 2} N g\left(-\frac{2}{|\lambda|} \tau\right)\right\rangle, \quad g \in S\left(\mathbb{C}^{n}\right),
\end{aligned}
$$

for $k \leq-p, \lambda \neq 0$, and

(2.3) $\left\langle F_{\lambda, k}, g\right\rangle$

$$
=\sum_{l=0}^{n-2}\left(\frac{2}{|\lambda|}\right)^{l} \frac{1}{|\lambda|} \sum_{\substack{l \leq j \leq n-2 \\
j \geq q-k-1}} \frac{(-1)^{n-j}}{2^{n+j-l}}\left(\begin{array}{c}
j \\
l
\end{array}\right)\left(\begin{array}{c}
n+k-q-1 \\
n-j-2
\end{array}\right)\left\langle\delta^{l}, N g\right\rangle, \quad g \in S\left(\mathbb{C}^{n}\right),
$$

for $-p<k<q, \lambda \neq 0$.

The next lemma states that (1.3) gives a relative fundamental solution $\Phi$ for $L$.

LEMma 2.1. The formula (1.3) defines a tempered distribution that satisfies $L(f * \Phi)=L(f) * \Phi=f-\pi(f)$ for all $f \in S\left(H_{n}\right)$.

Proof. We first prove that

$$
\sum_{2 k+p-q \neq 0} \frac{1}{|2 k+p-q|} \int_{-\infty}^{\infty}\left|\left\langle S_{\lambda, k}, f\right\rangle\right| \cdot|\lambda|^{n-1} d \lambda<\infty .
$$

Indeed, the above series can be written

$$
\begin{aligned}
\sum_{2 k+p-q \neq 0} & \frac{1}{|2 k+p-q|} \int_{-\infty}^{\infty}\left|\sum_{\|\alpha\|=k}\left\langle E_{\lambda}\left(h_{\alpha}, h_{\alpha}\right), f\right\rangle\right||\lambda|^{n-1} d \lambda \\
& =\sum_{2 k+p-q \neq 0} \frac{1}{|2 k+p-q|} \int_{-\infty}^{\infty}\left|\sum_{\|\alpha\|=k}\left\langle\pi_{\lambda}(f) h_{\alpha}, h_{\alpha}\right\rangle\right||\lambda|^{n-1} d \lambda \\
& \leq \sum_{k \in \mathbb{Z}^{n}} \int_{-\infty}^{\infty}\left|\left\langle\pi_{\lambda}(f) h_{\alpha}, h_{\alpha}\right\rangle\right| \cdot|\lambda|^{n-1} d \lambda .
\end{aligned}
$$

Now, proceeding as in [B-D-R], Lemma 4.10, we can use results of [M-R1] and [M-R2] to get (2.4) and so $\Phi$ is well defined. Moreover, the bounds given there show that $\Phi \in S^{\prime}\left(H_{n}\right)$. 
We recall that, for $f \in S\left(H_{n}\right), \pi f=0$ if $n$ is odd and that, for $n$ is even, $\pi f=\int_{-\infty}^{\infty}\left\langle S_{\lambda,(q-p) / 2}, f\right\rangle|\lambda|^{n} d \lambda$ (see [G-S], Remark 4.8).

In order to see that $L f * \Phi=f-\pi f$ we write (see, e.g., (1.3) in [G-S]) $L=L_{0}+L_{1}$ where

$$
\begin{aligned}
L_{0}= & \left(\sum_{j=1}^{p}\left(x_{j}^{2}+y_{j}^{2}\right)-\sum_{j=p+1}^{n}\left(x_{j}^{2}+y_{j}^{2}\right)\right) \frac{\partial^{2}}{\partial t^{2}} \\
& +\sum_{j=1}^{p}\left(\frac{\partial^{2}}{\partial x_{j}{ }^{2}}+\frac{\partial^{2}}{\partial y_{j}{ }^{2}}\right)-\sum_{j=p+1}^{n}\left(\frac{\partial^{2}}{\partial x_{j}{ }^{2}}+\frac{\partial^{2}}{\partial y_{j}{ }^{2}}\right)
\end{aligned}
$$

and

$$
L_{1}=\frac{\partial}{\partial t} \sum_{j=1}^{n}\left(x_{j} \frac{\partial}{\partial y_{j}}-y_{j} \frac{\partial}{\partial x_{j}}\right) .
$$

Now, for $g \in S\left(H_{n}\right)$ we have $L_{0}\left(g^{\vee}\right)=\left(L_{0}(g)\right)^{\vee}$ and $L_{1}\left(g^{\vee}\right)=-\left(L_{1}(g)\right)^{\vee}$. Since $(L f * \Phi)(z, t)=\left\langle\Phi,\left(L_{(z, t)^{-1}}(L f)\right)^{\vee}\right\rangle$ and $L=L_{0}+L_{1}$ we get

$$
(L f * \Phi)(z, t)=\left\langle\Phi, L_{0}\left(\left(L_{(z, t)^{-1}} f\right)^{\vee}\right)\right\rangle-\left\langle\Phi, L_{1}\left(\left(L_{(z, t)^{-1}} f\right)^{\vee}\right)\right\rangle .
$$

But $\Phi \in S^{\prime}\left(H_{n}\right)^{U(p, q)}$, and hence $L_{1} \Phi=0$. Also, $L_{0}$ and $L_{1}$ are self-adjoint, thus $(L f * \Phi)(z, t)=\left\langle\Phi, L\left(\left(L_{(z, t)^{-1}} f\right)^{\vee}\right)\right\rangle$. So

$$
\begin{aligned}
(L f * \Phi)(z, t) & =\sum_{2 k+p-q \neq 0} \frac{-1}{2 k+p-q} \int_{-\infty}^{\infty}\left\langle S_{\lambda, k}, L\left(\left(L_{(z, t)^{-1}} f\right)^{\vee}\right)\right\rangle|\lambda|^{n-1} d \lambda \\
& =\sum_{2 k+p-q \neq 0} \frac{-1}{2 k+p-q} \int_{-\infty}^{\infty}\left\langle L S_{\lambda, k},\left(L_{(z, t)^{-1}} f\right)^{\vee}\right\rangle|\lambda|^{n-1} d \lambda \\
& =\sum_{2 k+p-q \neq 0} \int_{-\infty}^{\infty}\left\langle S_{\lambda, k},\left(L_{(z, t)^{-1}} f\right)^{\vee}\right\rangle|\lambda|^{n} d \lambda \\
& =\sum_{2 k+p-q \neq 0} \int_{-\infty}^{\infty}\left(f * S_{\lambda, k}\right)(z, t)|\lambda|^{n} d \lambda=(f-\pi f)(z, t)
\end{aligned}
$$

and thus $L f * \Phi=f-\pi f$.

On the other hand, using (1.1), (1.2) and the fact that $L$ is a left invariant operator, it is easy to check that $L(f * \Phi)=f-\pi f$ for $f \in S\left(H_{n}\right)$.

Let us recall a well known Abel lemma about power series that states that if a numerical series $\sum_{k=0}^{\infty} a_{k}$ converges to a sum $s$, then $\lim _{r \rightarrow 1^{-}} \sum_{k=0}^{\infty} r^{k} a_{k}$ $=s$.

Taking account of (2.4), we can decompose the series (1.3) as

$$
\sum_{k \leq-p}+\sum_{-p<k<q}+\sum_{k \geq q}
$$


Now, (2.4), the Lebesgue dominated convergence theorem (applied to the measure space $\mathbb{R} \times \mathbb{Z}$ ) and the above Abel lemma allow us to write

$$
\begin{aligned}
\langle\Phi, f\rangle= & \lim _{r \rightarrow 1^{-}} \lim _{\varepsilon \rightarrow 0^{+}} \sum_{k \geq q} \frac{-r^{2 k+n-2 q}}{2 k+p-q} \int_{-\infty}^{\infty} e^{-\varepsilon|\lambda|}\left\langle S_{\lambda, k}, f\right\rangle|\lambda|^{n-1} d \lambda \\
& +\lim _{r \rightarrow 1^{-}} \lim _{\varepsilon \rightarrow 0^{+}} \sum_{k \leq-p} \frac{-r^{-2 k+n-2 p}}{2 k+p-q} \int_{-\infty}^{\infty} e^{-\varepsilon|\lambda|}\left\langle S_{\lambda, k}, f\right\rangle|\lambda|^{n-1} d \lambda \\
& +\lim _{\varepsilon \rightarrow 0^{+}} \sum_{-p<k<q} \frac{-1}{2 k+p-q} \int_{-\infty}^{\infty} e^{-\varepsilon|\lambda|}\left\langle S_{\lambda, k}, f\right\rangle|\lambda|^{n-1} d \lambda .
\end{aligned}
$$

We change the summation indices writing $k=k^{\prime}+q$ and $k=-k^{\prime}-p$ in the first and second series respectively. Using the fact that $S_{\lambda, k}=F_{\lambda, k} \otimes e^{-i \lambda t}$ and the formulas (2.1) and (2.2) we get

$$
\langle\Phi, f\rangle=\left\langle\Phi_{1}, f\right\rangle+\left\langle\Phi_{2}, f\right\rangle
$$

where $\left\langle\Phi_{1}, f\right\rangle$ and $\left\langle\Phi_{2}, f\right\rangle$ are defined by the convergent expressions

$$
\begin{aligned}
& \left\langle\Phi_{1}, f\right\rangle=\lim _{r \rightarrow 1^{-}} \lim _{\varepsilon \rightarrow 0^{+}} \sum_{k \geq 0} \frac{-r^{2 k+n}}{2 k+n} \int_{-\infty}^{\infty} \int_{-\infty}^{\infty} e^{-\varepsilon|\lambda|}|\lambda|^{n-1} e^{-i \lambda t} \\
& \times\left\langle\left(L_{k+n-1}^{0} H\right)^{(n-1)}, \tau \mapsto e^{-\tau / 2} \frac{2}{|\lambda|}\left[N f\left(\frac{2}{|\lambda|} \tau, t\right)-N f\left(-\frac{2}{|\lambda|} \tau, t\right)\right]\right\rangle d t d \lambda
\end{aligned}
$$

and

$$
\left\langle\Phi_{2}, f\right\rangle=\sum_{-p<k<q} \frac{-1}{2 k+p-q} \int_{-\infty}^{\infty}\left\langle S_{\lambda, k}, f\right\rangle|\lambda|^{n-1} d \lambda .
$$

Now, a computation gives

$$
\left(L_{k+n-1}^{0} H\right)^{(n-1)}=(-1)^{n-1} L_{k}^{n-1} H+\sum_{j=0}^{n-2}(-1)^{n-j}\left(\begin{array}{c}
k+n-1 \\
n-j-2
\end{array}\right) \delta^{(j)} .
$$

On the other hand,

$$
\begin{aligned}
\left\langle\delta^{(j)}, \tau \mapsto e^{-\tau / 2}\left[N f\left(\frac{2}{|\lambda|} \tau, t\right)-N f\left(-\frac{2}{|\lambda|} \tau, t\right)\right]\right\rangle & \\
= & -2 \sum_{\substack{0 \leq l \leq j \\
l \text { odd }}}\left(\begin{array}{l}
j \\
l
\end{array}\right) \frac{1}{2^{j-l}}\left(\frac{2}{|\lambda|}\right)^{l} \frac{\partial^{l} N f}{\partial \tau^{l}}(0, t) .
\end{aligned}
$$

Using (2.6), (2.7) and the fact that for $g \in L^{2}(0, \infty)$ and $h \in L^{2}(\mathbb{R})$,

$$
\int_{0}^{\infty} g(\tau)[h(\tau)-h(-\tau)] d \tau=\int_{-\infty}^{\infty} \operatorname{sgn}(\tau) g(|\tau|) h(\tau) d \tau
$$


we obtain

$$
\begin{gathered}
\left\langle\left(L_{k+n-1}^{0} H\right)^{(n-1)}, \tau \mapsto e^{-\tau / 2} \frac{2}{|\lambda|}\left[N f\left(\frac{2}{|\lambda|} \tau, t\right)-N f\left(-\frac{2}{|\lambda|} \tau, t\right)\right]\right\rangle \\
=(-1)^{n-1} \int_{-\infty}^{\infty} L_{k}^{n-1}\left(\frac{|\lambda|}{2}|s|\right) e^{-|\lambda| \cdot|s| / 4} \operatorname{sgn}(s) N f(s, t) d s \\
\quad-2 \sum_{\substack{0 \leq l \leq n-2 \\
l \text { odd }}}^{n-2} \sum_{j=l}^{n-2} b_{k, l}\left(\frac{2}{|\lambda|}\right)^{l+1} \frac{\partial^{l} N f}{\partial \tau^{l}}(0, t)
\end{gathered}
$$

where

$$
b_{k, l}=\sum_{j=l}^{n-2} \frac{1}{2^{j-l}}\left(\begin{array}{l}
j \\
l
\end{array}\right)(-1)^{n-j}\left(\begin{array}{l}
k+n-1 \\
n-j-2
\end{array}\right) .
$$

For $k \geq 0$ and $0 \leq l \leq n-2$, we also set

$$
a_{k, l}=\frac{1}{l !} \int_{0}^{\infty} L_{k}^{n-1}(s) e^{-s / 2} s^{l} d s
$$

and

$$
G_{f}(\tau, t)=N f(\tau, t)-\sum_{j=0}^{n-2} \frac{\partial^{l} N f}{\partial \tau^{j}}(0, t) \frac{\tau^{j}}{j !} .
$$

Let $\Phi_{1,1}$ be the linear functional on $S\left(H_{n}\right)$ defined by

$$
\begin{aligned}
\left\langle\Phi_{1,1}, f\right\rangle= & \lim _{r \rightarrow 1^{-}} \sum_{k \geq 0} \frac{(-1)^{n} r^{2 k+n}}{2 k+n} \lim _{\varepsilon \rightarrow 0^{+}} \int_{-\infty}^{\infty} \int_{-\infty}^{\infty} e^{-\varepsilon|\lambda|}|\lambda|^{n-1} e^{-i \lambda t} \\
& \times \int_{-\infty}^{\infty} L_{k}^{n-1}\left(\frac{|\lambda|}{2}|\tau|\right) e^{-|\lambda| \cdot|\tau| / 4} \operatorname{sgn}(\tau) G_{f}(\tau, t) d \tau d t d \lambda .
\end{aligned}
$$

We will prove below (Proposition 4.3) that $\left\langle\Phi_{1,1}, f\right\rangle$ makes sense, i.e. that the expression (2.12) converges. Assume provisionally this fact. From (2.5) it follows that $\Phi_{1,2}: S\left(H_{n}\right) \rightarrow \mathbb{C}$ defined by

$$
\begin{aligned}
\left\langle\Phi_{1,2}, f\right\rangle= & 2 \lim _{r \rightarrow 1^{-}} \lim _{\varepsilon \rightarrow 0^{+}} \sum_{k \geq 0} \frac{r^{2 k+n}}{2 k+n} \int_{-\infty}^{\infty} \int_{-\infty}^{\infty} e^{-\varepsilon|\lambda|}|\lambda|^{n-1} e^{-i \lambda t} \\
& \times \sum_{\substack{0 \leq l \leq n-2 \\
l \text { odd }}}\left(\frac{2}{|\lambda|}\right)^{l+1}\left(a_{k, l}+b_{k, l}\right) \frac{\partial^{l} N f}{\partial \tau^{l}}(0, t) d t d \lambda
\end{aligned}
$$

is also well defined and that $\Phi_{1}=\Phi_{1,1}+\Phi_{1,2}$ and so $\Phi=\Phi_{1,1}+\Phi_{1,2}+\Phi_{2}$.

Note that the same arguments that prove that $N: S^{\prime}\left(\mathbb{C}^{n}\right) \rightarrow \mathcal{H}$ is surjective, show that for $f \in S\left(H_{n}\right), N f(\tau, t)$ agrees with a certain function 
$\varphi_{1} \in S\left(\mathbb{R}^{2}\right)$ on $\left\{(\tau, t) \in \mathbb{R}^{2}: \tau>0\right\}$ and with another function $\varphi_{2} \in S\left(\mathbb{R}^{2}\right)$ on $\left\{(\tau, t) \in \mathbb{R}^{2}: \tau<0\right\}$. So, for each polynomial $P(\tau, t)$ and for each pair of nonnegative integers $\alpha=\left(\alpha_{1}, \alpha_{2}\right)$ we have

$$
\sup _{\tau \neq 0, t \in \mathbb{R}}\left|P(\tau, t) D^{\alpha} N f(\tau, t)\right|<\infty .
$$

In order to see that $\Phi_{1,1}$ is well defined, we first note that, for $k \in \mathbb{Z}$ and $\varepsilon>0,(2.14)$ implies that the function

$$
\operatorname{sgn}(\tau) e^{-\varepsilon|\lambda|-i \lambda t}|\lambda|^{n-1} L_{k}^{n-1}\left(\frac{|\lambda|}{2}|\tau|\right) e^{-|\lambda| \cdot|\tau| / 4} G_{f}(\tau, t)
$$

belongs to $L^{1}\left(\mathbb{R}^{3}, d \tau d t d \lambda\right)$. On the other hand, by (2.8),

$$
\begin{aligned}
& \int_{-\infty}^{\infty} e^{-\varepsilon|\lambda|-i \lambda t} L_{k}^{n-1}\left(\frac{|\lambda|}{2}|\tau|\right) e^{-|\lambda| \cdot|\tau| / 4}|\lambda|^{n-1} d \lambda \\
& =2 \operatorname{Re} \int_{0}^{\infty} e^{-\varepsilon \lambda-i \lambda t} L_{k}^{n-1}\left(\frac{\lambda}{2}|\tau|\right) e^{-\lambda|\tau| / 4} \lambda^{n-1} d \lambda
\end{aligned}
$$

and as in (4.9) of [G-S], writing there $\tau$ instead of $B(z)$, we find that the value of this integral is

$$
2 \alpha_{k} \operatorname{Re}\left(\frac{(|\tau|-4 \varepsilon-4 i t)^{k}}{(|\tau|+4 \varepsilon+4 i t)^{k+n}}\right)
$$

with

$$
\alpha_{k}=4^{n}(n-1) !(-1)^{k}\left(\begin{array}{c}
k+n-1 \\
k
\end{array}\right) .
$$

Thus, to prove that (2.12) converges, we must show the convergence of

$$
\begin{aligned}
\lim _{r \rightarrow 1^{-}} \sum_{k \geq 0}(-1)^{n} \frac{r^{2 k+n}}{2 k+n} \lim _{\varepsilon \rightarrow 0^{+}} 2 \alpha_{k} \int_{\mathbb{R}^{2}} \operatorname{Re}\left(\frac{(|\tau|-4 \varepsilon-4 i t)^{k}}{(|\tau|+4 \varepsilon+4 i t)^{k+n}}\right) \\
\times \operatorname{sgn}(\tau) G_{f}(\tau, t) d \tau d t .
\end{aligned}
$$

We will need the following

Lemma 2.2. The function

$$
\frac{1}{\left(\tau^{2}+16 t^{2}\right)^{n / 2}} G_{f}(\tau, t)
$$

is integrable on $\mathbb{R}^{2}$.

Proof. Let $B=\left\{(\tau, t): \tau^{2}+16 t^{2}<1\right\}$. From (2.14) and the familiar expression for the remainder of a Taylor development, it follows that there exists a positive constant $c$ such that for $(\tau, t) \in B$,

$$
\left|\frac{1}{\left(\tau^{2}+16 t^{2}\right)^{n / 2}} G_{f}(\tau, t)\right| \leq \frac{c}{\left(|\tau|^{2}+16 t^{2}\right)^{n / 2}}|\tau|^{n-1}
$$


and a change to polar coordinates shows that $|\tau|^{n-1}\left(|\tau|^{2}+16 t^{2}\right)^{-n / 2}$ is integrable on $B$.

On the other hand, for $(\tau, t) \in \mathbb{R}^{2}-B$ we have

$$
\begin{aligned}
& \left|\frac{1}{\left(\tau^{2}+16 t^{2}\right)^{n / 2}} G_{f}(\tau, t)\right| \\
& \leq\left[|N f(\tau, t)|+\sum_{j=1}^{n-2} \frac{|\tau|^{j}}{j !}\left|\frac{\partial^{j} N f}{\partial \tau^{j}}(0, t)\right|\right] \frac{1}{\left(|\tau|^{2}+16 t^{2}\right)^{n / 2}} .
\end{aligned}
$$

Let $A=\{(\tau, t):|t| \leq 1 / 2\} \cap\left(\mathbb{R}^{2}-B\right)$. For $(\tau, t) \in A$, we have $|\tau| \geq 1 / 2$ and so, by (2.14), for $1 \leq j \leq n-2$ we have

$$
\int_{A}\left|\frac{\partial^{j} N f}{\partial \tau^{j}}(0, t)\right| \frac{|\tau|^{j}}{\left(|\tau|^{2}+16 t^{2}\right)^{n / 2}} d \tau d t \leq \int_{-1 / 2}^{1 / 2}\left|\frac{\partial^{j} N f}{\partial \tau^{j}}(0, t)\right| d t \int_{1 / 2}^{\infty} \frac{1}{\tau^{2}} d \tau<\infty .
$$

The same argument shows that the analogous integral with $\partial^{j} N f(0, t) / \partial \tau^{j}$ replaced by $N f(\tau, t)$ is finite. Now, let $A^{\prime}=\{(\tau, t):|t|>1 / 2\} \cap\left(\mathbb{R}^{2}-B\right)$. For $(\tau, t) \in A^{\prime}$ and $0 \leq j \leq n-2$ we have

$$
|\tau|^{j}\left(|\tau|^{2}+16 t^{2}\right)^{-n / 2} \leq|\tau|^{j}\left(|\tau|^{2}+4\right)^{-n / 2}
$$

and by (2.14), $\left|G_{f}(\tau, t)\right| \leq c\left(1+t^{2}\right)^{-1}$. Thus

$$
\int_{A^{\prime}}\left(|\tau|^{2}+16 t^{2}\right)^{-n / 2}\left|G_{f}(\tau, t)\right| d \tau d t<\infty
$$

\section{LEMMA 2.3.}

$$
\begin{aligned}
\lim _{\varepsilon \rightarrow 0^{+}} & \int_{\mathbb{R}^{2}} \operatorname{Re}\left(\frac{(|\tau|-4 \varepsilon-4 i t)^{k}}{(|\tau|+4 \varepsilon+4 i t)^{k+n}}\right) \operatorname{sgn}(\tau) G_{f}(\tau, t) d \tau d t \\
\quad & \int_{\mathbb{R}^{2}} \frac{1}{\left(\tau^{2}+16 t^{2}\right)^{n / 2}} \operatorname{Re}\left(\left(\frac{|\tau|-4 i t}{\left(\tau^{2}+16 t^{2}\right)^{1 / 2}}\right)^{2 k+n}\right) \operatorname{sgn}(\tau) G_{f}(\tau, t) d \tau d t .
\end{aligned}
$$

Proof. This follows from Lemma 2.2 and the Lebesgue dominated convergence theorem.

So, to see that $\left\langle\Phi_{1,1}, f\right\rangle$ is well defined for $f \in S\left(H_{n}\right)$, we need to study the convergence of

$$
\begin{aligned}
\lim _{r \rightarrow 1^{-}} \sum_{k \geq 0}(-1)^{n} \frac{r^{2 k+n}}{2 k+n} \alpha_{k} \int_{\mathbb{R}^{2}} \frac{1}{\left(\tau^{2}+16 t^{2}\right)^{n / 2}} \\
\times 2 \operatorname{Re}\left(\left(\frac{|\tau|-4 i t}{\left(\tau^{2}+16 t^{2}\right)^{1 / 2}}\right)^{2 k+n}\right) \operatorname{sgn}(\tau) G_{f}(\tau, t) d \tau d t
\end{aligned}
$$

with $\alpha_{k}$ given by (2.15). We decompose this integral as the sum of integrals over the right and left halfplanes. Taking polar coordinates $\tau-4 i t=\varrho e^{i \theta}$, 
we can see that the above expression agrees with

$$
2(-1)^{n} \lim _{r \rightarrow 1^{-}} \operatorname{Re}\left(\sum_{k \geq 0} \frac{r^{2 k+n}}{2 k+n} \alpha_{k} \int_{0}^{\infty} \int_{0}^{2 \pi} e^{i(2 k+n) \theta} K_{f}(\varrho, \theta) d \theta d \varrho\right)
$$

where $K_{f}(\varrho, \theta)$ is the function defined for $n$ odd by

$$
K_{f}(\varrho, \theta)=\varrho^{1-n} G_{f}\left(\varrho \cos (\theta),-\frac{1}{4} \varrho \sin (\theta)\right)
$$

and for $n$ even by

$$
K_{f}(\varrho, \theta)=\operatorname{sgn}(\cos (\theta)) \varrho^{1-n} G_{f}\left(\varrho \cos (\theta),-\frac{1}{4} \varrho \sin (\theta)\right) .
$$

Note that, for $0<r<1$, from Lemma 2.2, it follows that

$$
\sum_{k \geq 0} \frac{r^{2 k+n}}{2 k+n}\left|\alpha_{k}\right| \int_{0}^{\infty} \int_{0}^{2 \pi}\left|K_{f}(\varrho, \theta)\right| d \varrho d \theta<\infty,
$$

so $(2.17)$ can be written as

$$
2(-1)^{n} \lim _{r \rightarrow 1^{-}} \operatorname{Re} \int_{0}^{\infty}\left[\sum_{k \geq 0} \frac{r^{2 k+n}}{2 k+n} \alpha_{k} \int_{0}^{2 \pi} e^{i(2 k+n) \theta} K_{f}(\varrho, \theta) d \theta\right] d \varrho .
$$

Recall that $\alpha_{k}=4^{n}(n-1) !(-1)^{k}\left(\begin{array}{c}k+n-1 \\ k\end{array}\right)$. So, in order to study $(2.20)$, in the next section we will study the distributions defined, for $0 \leq r<1$ and $\theta \in \mathbb{R}$, by

$$
\Psi_{r}(\theta)=\operatorname{Re}\left[\sum_{k \geq 0} \frac{r^{2 k+n}}{2 k+n}(-1)^{k}\left(\begin{array}{c}
k+n-1 \\
k
\end{array}\right) e^{i(2 k+n) \theta}\right] .
$$

3. Computation of $\lim _{r \rightarrow 1^{-}} \Psi_{r}$. Let $\Psi_{r}$ be defined by (2.21). Thus

$$
\frac{d \Psi_{r}}{d \theta}=\operatorname{Re}\left[i \sum_{k \geq 0} r^{2 k+n}(-1)^{k}\left(\begin{array}{c}
k+n-1 \\
k
\end{array}\right) e^{i(2 k+n) \theta}\right] .
$$

Now, we take $t=0, z=-r^{2} e^{2 i \theta}$ in the generating identity for the Laguerre polynomials

$$
\sum_{k \geq 0} L_{k}^{n-1}(t) z^{k}=(1-z)^{-n} e^{-z t /(1-z)} .
$$

Using the fact that $L_{k}^{n-1}(0)=\left(\begin{array}{c}k+n-1 \\ k\end{array}\right)$ we obtain

$$
\frac{d \Psi_{r}}{d \theta}=-\operatorname{Im}\left(\left(\frac{r e^{i \theta}}{1+r^{2} e^{2 i \theta}}\right)^{n}\right) .
$$


Note that the limit $\lim _{r \rightarrow 1^{-}} \Psi_{r}(0)$ exists. Indeed,

$$
\Psi_{r}(0)=\sum_{k \geq 0} \frac{r^{2 k+n}}{2 k+n}(-1)^{k}\left(\begin{array}{c}
k+n-1 \\
k
\end{array}\right) \quad \text { for } 0 \leq r<1 .
$$

So, a computation shows that $d \Psi_{r}(0) / d r=r^{n-1} /\left(1+r^{2}\right)^{n}$ for $0 \leq r<1$. Also, $\Psi_{0}(0)=0$ and thus

$$
\lim _{r \rightarrow 1^{-}} \Psi_{r}(0)=\int_{0}^{1} \sigma^{n-1}\left(1+\sigma^{2}\right)^{-n} d \sigma .
$$

We also have

Proposition 3.1. If $0<\delta<\pi / 2$ then there exists a positive constant c such that $\left|\Psi_{r}(\theta)-\Psi_{r}(0)\right| \leq c \theta^{2}(1-r)$ for $-\delta<\theta<\delta$ and $0<r<1$.

Proof. From (3.3), we have

$$
\left|\Psi_{r}(\theta)-\Psi_{r}(0)\right| \leq \int_{0}^{\theta}\left|\operatorname{Im}\left(\left(\frac{r e^{i \sigma}}{1+r^{2} e^{2 i \sigma}}\right)^{n}\right)\right| d \sigma .
$$

Now,

$$
\begin{aligned}
& \operatorname{Im}\left(\left(\frac{r e^{i \sigma}}{1+r^{2} e^{2 i \sigma}}\right)^{n}\right)=\frac{\operatorname{Im}\left(r^{n} e^{i n \sigma}\left(1+r^{2} e^{-2 i \sigma}\right)^{n}\right)}{\left(1+2 r^{2} \cos (2 \sigma)+r^{4}\right)^{n}} \\
& =r^{n} \frac{\sin (n \sigma) \sum_{j=0}^{n}\left(\begin{array}{c}
n \\
j
\end{array}\right) r^{2 j} \cos (2 j \sigma)-\cos (n \sigma) \sum_{j=1}^{n}\left(\begin{array}{c}
n \\
j
\end{array}\right) r^{2 j} \sin (2 j \sigma)}{\left(1+2 r^{2} \cos (2 \sigma)+r^{4}\right)^{n}} .
\end{aligned}
$$

An induction on $n$ shows that $\operatorname{Im}\left(\left(\frac{e^{i \sigma}}{1+e^{2 i \sigma}}\right)^{n}\right)=0$ for $\sigma \notin \pi / 2+\pi \mathbb{Z}$. Also, the denominator of (3.6) is bounded away from 0 , and so the numerator is a polynomial expression in $r$ that vanishes at $r=1$ for all $\sigma$ whose coefficients are trigonometrical polynomials divisible by $\sin (\sigma)$. So the numerator can be written as $(1-r) \sin (\sigma) Q(r, \cos (\sigma), \sin (\sigma)))$ where $Q$ is a polynomial in its arguments. It follows that there exists a positive constant $c$ such that

$$
\left|\operatorname{Im}\left(\left(\frac{r e^{i \sigma}}{1+r^{2} e^{2 i \sigma}}\right)^{n}\right)\right| \leq c|\sin (\sigma)|(1-r)
$$

for $0 \leq r<1$ and $|\theta|<\delta$ and so (3.5) gives the lemma.

REMARK 3.2. If $0<\delta<\pi / 2$, the same argument shows that $\mid \Psi_{r}(\theta)-$ $\Psi_{r}(\pi) \mid \leq$ const $(\theta-\pi)^{2}(1-r)$ for $-\delta<\theta-\pi<\delta$ and $0<r<1$.

Let $\mathcal{V}$ be the vector space of functions $g \in C^{n-2}\left(S^{1}\right)$ satisfying $(\partial / \partial \theta)^{n-1} g$ $\in L^{\infty}\left(S^{1}\right)$. For $h: S^{1} \rightarrow \mathbb{C}$ and $g \in \mathcal{V}$ we write $\langle h, g\rangle$ for $\int_{0}^{2 \pi} h\left(e^{i \theta}\right) g\left(e^{i \theta}\right) d \theta$ whenever the integral makes sense. 
For $g \in \mathcal{V}$ let

$$
\langle\Psi, g\rangle=\operatorname{Re} \sum_{k \geq 0} \frac{1}{2 k+n}(-1)^{k}\left(\begin{array}{c}
k+n-1 \\
k
\end{array}\right) \int_{-\pi}^{\pi} e^{i(2 k+n) \theta} g\left(e^{i \theta}\right) d \theta .
$$

Let $\widetilde{H}: S^{1} \rightarrow \mathbb{R}$ be the function defined by $\widetilde{H}\left(e^{i \theta}\right)=H(\cos (\theta))$. Also, for $s \in \mathbb{R}$, let $\delta_{s}: \mathcal{V} \rightarrow \mathbb{R}$ be the distribution given by $\left\langle\delta_{s}, g\right\rangle=g\left(e^{i s}\right)$.

Proposition 3.3. For all $g \in \mathcal{V}$ the series (3.7) converges. Moreover:

(1) If $n$ is even, then there exists a constant $c_{0}$ and a polynomial $Q_{n-2}$ of degree $n-2$ such that for all $g \in \mathcal{V}$,

$$
\langle\Psi, g\rangle=-c_{0}\langle 1, g\rangle+\left\langle Q_{n-2}\left(\frac{\partial}{\partial \theta}\right)\left(\delta_{\pi / 2}+\delta_{-\pi / 2}\right), g\right\rangle .
$$

(2) If $n$ is odd, then there exist two constants $c_{0}$ and $d_{0}$ and a polynomial $Q_{n-2}$ of degree $n-2$ such that for all $g \in \mathcal{V}$,

$$
\langle\Psi, g\rangle=\left\langle-c_{0}+d_{0} \widetilde{H}, g\right\rangle+\left\langle Q_{n-2}\left(\frac{\partial}{\partial \theta}\right)\left(\delta_{\pi / 2}-\delta_{-\pi / 2}\right), g\right\rangle .
$$

Proof. For $g \in \mathcal{V}$, the convergence of (3.7) follows from the fact that $\frac{1}{2 k+n}\left(\begin{array}{c}k+n-1 \\ k\end{array}\right)=O\left(k^{n-2}\right)$ and $\left|\left\langle g, e^{i \kappa \theta}\right\rangle\right| \leq|k|^{-(n-1)}\left|\left\langle g^{(n-1)}, e^{i \kappa \theta}\right\rangle\right|$ with $g^{(n-1)} \in L^{2}\left(S^{1}\right)$.

The familiar Fourier expansion for a function $g \in \mathcal{V}$ can be read as $\delta_{s}=\sum_{k \in \mathbb{Z}} e^{i k s} \phi_{k}$, where $\phi_{k}\left(e^{i \theta}\right)=e^{i k \theta}$. Then a computation gives

$$
\frac{1}{2}\left(\delta_{\pi / 2}+\delta_{-\pi / 2}\right)=\sum_{j \in \mathbb{Z}}(-1)^{j} e^{i 2 j \theta}, \quad \frac{1}{2}\left(\delta_{\pi / 2}-\delta_{-\pi / 2}\right)=\sum_{j \in \mathbb{Z}}(-1)^{j} e^{i(2 j+1) \theta} .
$$

From (3.7) we have

$$
\Psi^{\prime}=\operatorname{Re} \sum_{k \geq 0}(-1)^{k} \frac{(k+n-1) \ldots(k+1)}{(n-1) !} i e^{i(2 k+n) \theta} .
$$

Assume $n$ is even. A change of the summation index in (3.8) gives

$$
\Psi^{\prime}=\operatorname{Re} \sum_{j \geq n / 2}(-1)^{j+n / 2} \frac{(2 j-n+2) \ldots(2 j+n-2)}{2^{n-1}(n-1) !} i e^{i 2 j \theta} .
$$

On the other hand, the change $2 k+n=-2 j$ of the summation index in (3.7) also gives

$$
\Psi^{\prime}=\operatorname{Re} \sum_{j \leq n / 2}(-1)^{j+n / 2} \frac{(2 j-n+2) \ldots(2 j+n-2)}{2^{n-1}(n-1) !} i e^{i 2 j \theta}
$$


and, since $n$ is even, we also have $(2 j+n-2) \ldots(2 j-n+2)=0$ for $-n / 2<j<n / 2$. Thus

$$
\Psi^{\prime}=\frac{1}{2} \operatorname{Re} \sum_{k \in \mathbb{Z}}(-1)^{k+n / 2} \frac{(2 k-n+2) \ldots(2 k+n-2)}{2^{n-1}(n-1) !} i e^{i 2 k \theta} .
$$

Let $P_{n-1}$ the polynomial of degree $n-1$ given by

$$
P_{n-1}(s)=\frac{(2 s-n+2) \ldots(2 s+n-2)}{2^{n}(n-1) !}, \quad s \in \mathbb{R},
$$

and let $\Theta=\sum_{k \in \mathbb{Z}}(-1)^{k+n / 2} P_{n-1}(k) i e^{i 2 k \theta}$. Thus $\Psi^{\prime}=\operatorname{Re} \Theta$. Since $n$ is even, we have $P_{n-1}(0)=0$ and so $P_{n-1}(s)=s \widetilde{Q}_{n-2}(s)$ for some polynomial $\widetilde{Q}_{n-2}$ of degree $n-2$. Now, $k^{l} e^{i 2 k \theta}=\left(\frac{1}{2 i} \frac{\partial}{\partial \theta}\right)^{l} e^{i 2 k \theta}, l \geq 0$, and so

$$
\Theta=\frac{1}{2 i} \frac{\partial}{\partial \theta}\left(i \sum_{k \in \mathbb{Z}}(-1)^{k+n / 2} \widetilde{Q}_{n-2}\left(\frac{1}{2 i} \frac{\partial}{\partial \theta}\right) e^{i 2 k \theta}\right) .
$$

Then, taking account of $\frac{1}{2}\left(\delta_{\pi / 2}+\delta_{-\pi / 2}\right)=\sum_{k \in \mathbb{Z}}(-1)^{k} e^{i 2 k \theta}$, we obtain

$$
\left\langle\Psi^{\prime}, g\right\rangle=\operatorname{Re}\langle\Theta, g\rangle=\left\langle\frac{\partial}{\partial \theta} Q_{n-2}\left(\frac{\partial}{\partial \theta}\right)\left(\delta_{\pi / 2}+\delta_{-\pi / 2}\right), g\right\rangle
$$

for some polynomial $Q_{n-2}$ of degree $n-2$ with real coefficients. So

$$
\Psi=-c_{0}+Q_{n-2}\left(\frac{\partial}{\partial \theta}\right)\left(\delta_{\pi / 2}+\delta_{-\pi / 2}\right)
$$

for some constant $c_{0}$. This ends the proof for the case of $n$ even.

Assume now that $n$ is odd. By (3.7) we now have

$$
\Psi^{\prime}=\operatorname{Re} \sum_{2 j+1 \geq n}(-1)^{j+(n-1) / 2} \frac{(2 j+n-1) \ldots(2 j-n+3)}{2^{n-1}(n-1) !} i e^{i(2 j+1) \theta} .
$$

Thus we get, as above,

$$
\Psi^{\prime}=\frac{1}{2} \operatorname{Re} \sum_{k \in \mathbb{Z}}(-1)^{j+(n-1) / 2} \frac{(2 j+n-1) \ldots(2 j-n+3)}{2^{n-1}(n-1) !} i e^{i(2 j+1) \theta} .
$$

Let $P_{n-1}$ be the polynomial given by

$$
P_{n-1}(2 s+1)=\frac{(2 s+n-1) \ldots(2 s-n+3)}{2^{n}(n-1) !}, \quad s \in \mathbb{R},
$$

and let $\Theta=\sum_{k \in \mathbb{Z}}(-1)^{k+(n-1) / 2} P_{n-1}(2 k+1) i e^{i(2 k+1) \theta}$. Thus $\Psi^{\prime}=\operatorname{Re} \Theta$. Now

$$
\Theta=\sum_{k \in \mathbb{Z}}(-1)^{k+(n-1) / 2} P_{n-1}\left(\frac{1}{2 i} \frac{\partial}{\partial \theta}\right) i e^{i 2 k \theta} .
$$


But $\frac{1}{2}\left(\delta_{\pi / 2}-\delta_{-\pi / 2}\right)=\sum_{k \in \mathbb{Z}}(-1)^{k} e^{i(2 k+1) \theta}$ and hence

$$
\left\langle\Psi^{\prime}, g\right\rangle=\operatorname{Re}\langle\Theta, g\rangle=\left\langle\widetilde{P}_{n-1}\left(\frac{\partial}{\partial \theta}\right)\left(\delta_{\pi / 2}-\delta_{-\pi / 2}\right), g\right\rangle
$$

for some polynomial $\widetilde{P}_{n-1}$ of degree $n-1$ with real coefficients. Thus,

$$
\left\langle\Psi^{\prime}, g\right\rangle=-d_{0}\left(\delta_{\pi / 2}-\delta_{-\pi / 2}\right)+\frac{\partial}{\partial \theta} Q_{n-2}\left(\frac{\partial}{\partial \theta}\right)\left(\delta_{\pi / 2}-\delta_{-\pi / 2}\right)
$$

for some polynomial $Q_{n-2}$ of degree $n-2$ and some constant $d_{0}$. Now, $\delta_{\pi / 2}-\delta_{-\pi / 2}=-\frac{\partial}{\partial \theta} \widetilde{H}$, and so

$$
\Psi^{\prime}=\frac{\partial}{\partial \theta} Q_{n-2}\left(\frac{\partial}{\partial \theta}\right)\left(\delta_{\pi / 2}-\delta_{-\pi / 2}\right)+d_{0} \frac{\partial}{\partial \theta} \tilde{H}
$$

Thus $\Psi=-c_{0}+d_{0} \widetilde{H}+Q_{n-2}(\partial / \partial \theta)\left(\delta_{\pi / 2}-\delta_{-\pi / 2}\right)$ for some constant $c_{0}$.

Corollary 3.4. $\lim _{r \rightarrow 1^{-}}\left\langle\Psi_{r}, g\right\rangle=\langle\Psi, g\rangle$ for all $g \in \mathcal{V}$.

Proof. Follows from the Abel lemma and Proposition 3.3.

Proposition 3.5. If $n$ is even and if $c_{0}$ is the constant of Proposition 3.3 then $c_{0}=-\lim _{r \rightarrow 1^{-}} \Psi_{r}(0)$.

If $n$ is odd and if $c_{0}, d_{0}$ are the constants of Proposition 3.3 then $c_{0}=$ $\lim _{r \rightarrow 1^{-}} \Psi_{r}(0)$ and $d_{0}=2 c_{0}$.

Proof. Assume that $n$ is odd. Let $h \in C_{\mathrm{c}}^{\infty}(\mathbb{R})$ be such that $\operatorname{supp}(h) \subset$ $(-\pi / 4, \pi / 4), h \geq 0$ and $\int h=1$, and let $Q_{n-2}$ be the polynomial given by Proposition 3.3. Thus $Q_{n-2}(\partial / \partial \theta)\left(\delta_{\pi / 2} \pm \delta_{-\pi / 2}\right)(h)=0$ and so

$$
\lim _{r \rightarrow 1^{-}}\left\langle\Psi_{r}, h\right\rangle=\left\langle-c_{0}+d_{0} \widetilde{H}, h\right\rangle=-c_{0}+d_{0} .
$$

On the other hand, since $\int h=1$ we have

$$
\lim _{r \rightarrow 1^{-}}\left\langle\Psi_{r}, h\right\rangle=\lim _{r \rightarrow 1^{-}}\left[\int\left(\Psi_{r}(\theta)-\Psi_{r}(0)\right) h(\theta) d \theta+\Psi_{r}(0)\right]
$$

and by Proposition 3.1,

$$
\begin{aligned}
& \left|\int\left(\Psi_{r}(\theta)-\Psi_{r}(0)\right) h(\theta) d \theta\right| \leq\left|\int_{|\theta|<1 / 2}\right| \Psi_{r}(\theta)-\Psi_{r}(0)|h(\theta) d \theta| \\
& \leq \operatorname{const}(1-r)\left|\int_{|\theta|<1 / 2} \theta^{2} h(\theta) d \theta\right| \leq \operatorname{const}(1-r)
\end{aligned}
$$

and so $-c_{0}+d_{0}=\lim _{r \rightarrow 1^{-}} \Psi_{r}(0)$. Similarly, taking $h \in C_{\mathrm{c}}^{\infty}(\mathbb{R})$ such that $\operatorname{supp}(h) \subset(\pi / 2,3 \pi / 2)$ with $h \geq 0$ and $\int h=1$ we get $d_{0}=\lim _{r \rightarrow 1^{-}} \Psi_{r}(\pi)$. Since $\Psi_{r}(\pi)=-\Psi_{r}(0)$ if $n$ is odd, the lemma follows in this case. The proof for $n$ even follows the same lines. 


\section{The main result}

Remark 4.1. Let $K_{f}(\varrho, \theta)$ be defined by (2.18) and (2.19). A computation shows that $K_{f}(\varrho, \cdot) \in C^{n-2}(\mathbb{R})$ for each $\varrho \in(0, \infty)$. Moreover, for such a $\varrho, d^{n-1} K_{f}(\varrho, \theta) / d \theta^{n-1}$ is well defined for $\theta \notin \pi / 2+\mathbb{Z}$,

$$
\frac{d^{j} K_{f}(\varrho, \cdot)}{d \theta^{j}}\left( \pm \frac{\pi}{2}\right)=0 \quad \text { for } j=0, \ldots, n-2
$$

and $d^{n-1} K_{f}(\varrho, \theta) / d \theta^{n-1} \in L^{\infty}(\mathbb{R})$. We also need the following facts.

Let $A=[\delta, \pi-\delta] \cup[\pi+\delta, 2 \pi-\delta]$ with $0<\delta<\pi / 2$ and let $\widetilde{K}_{f}: A \rightarrow \mathbb{R}$ be defined by $\widetilde{K}_{f}(\theta)=\int_{1}^{\infty} K_{f}(\varrho, \theta) d \varrho$. Then a computation using $(2.14)$ and the Lebesgue dominated convergence theorem show that $\widetilde{K}_{f} \in C^{n-1}(A)$. Moreover $\widetilde{K}_{f}^{(j)}( \pm \pi / 2)=0$ for $0 \leq j \leq n-2$. The same argument gives that the function $\int_{0}^{1} K_{f}(\varrho, \cdot) d \varrho$ belongs to $\mathcal{V}$ with its $n-2$ first derivatives vanishing at $\pm \pi / 2$.

Proposition 4.2. Let $\alpha_{k}$ be given by (2.15) and let $c_{0}$ be as in Proposition 3.3. Then

$$
\begin{aligned}
\lim _{r \rightarrow 1^{-}} \operatorname{Re} \int_{0}^{\infty} & {\left[\sum_{k \geq 0} \frac{r^{2 k+n}}{2 k+n} \alpha_{k} \int_{0}^{2 \pi} e^{i(2 k+n) \theta} K_{f}(\varrho, \theta) d \theta\right] d \varrho } \\
& =4^{n}(n-1) ! c_{0} \int_{\mathbb{R}^{2}} \frac{1}{\left(\tau^{2}+16 t^{2}\right)^{n / 2}} \operatorname{sgn}(\tau) G_{f}(\tau, t) d \tau d t .
\end{aligned}
$$

Proof. Assume $n$ is odd. Since

$$
\begin{aligned}
& c_{0} \int_{\mathbb{R}^{2}} \frac{1}{\left(\tau^{2}+16 t^{2}\right)^{n / 2}} G_{f}(\tau, t) d \tau d t \\
& \quad=\int_{\mathbb{R}^{2}} \frac{1}{\left(\tau^{2}+16 t^{2}\right)^{n / 2}}\left(-c_{0}+d_{0} H(\tau)\right) \operatorname{sgn}(\tau) G_{f}(\tau, t) d \tau d t \\
& \quad=\int_{0}^{\infty} \int_{0}^{2 \pi}\left(-c_{0}+d_{0} \widetilde{H}(\theta)\right) K_{f}(\varrho, \theta) d \theta d \varrho
\end{aligned}
$$

it is enough to prove that

$$
\lim _{r \rightarrow 1^{-}} \int_{0}^{\infty}\left\langle\Psi_{r}, K_{f}(\varrho, \cdot)\right\rangle d \varrho=\int_{0}^{\infty}\left\langle-c_{0}+d_{0} \widetilde{H}, K_{f}(\varrho, \cdot)\right\rangle d \varrho .
$$

In order to see this, we decompose the integral as $\int_{0}^{1}+\int_{1}^{\infty}$. To study the second term of this sum, we pick $0<\delta<\pi / 4$. We first show that

$$
\lim _{r \rightarrow 1^{-}} \int_{1}^{\infty} \int_{|\theta|<\delta}\left(\Psi_{r}(\theta)-\left(-c_{0}+d_{0} \widetilde{H}(\theta)\right)\right) K_{f}(\varrho, \theta) d \theta d \varrho=0 .
$$


To see this, we decompose the integral in (4.3) as

$$
\begin{aligned}
& \int_{1}^{\infty} \int_{|\theta|<\delta}\left(\Psi_{r}(\theta)-\Psi_{r}(0)\right) K_{f}(\varrho, \theta) d \theta d \varrho \\
& +\left(\Psi_{r}(0)-\left(-c_{0}+d_{0}\right)\right) \int_{1}^{\infty} \int_{|\theta|<\delta} K_{f}(\varrho, \theta) d \theta d \varrho .
\end{aligned}
$$

From the definition of $K_{f}(\varrho, \theta)$ and $(2.14)$ we have $\left|K_{f}(\varrho, \theta)\right| \leq c \varrho^{-2}|\sin (\theta)|^{-1}$ for some constant $c$ and for all $\varrho>1,|\theta|<\delta$. Then by Proposition 3.1,

$$
\begin{aligned}
& \left|\int_{1}^{\infty} \int_{|\theta|<\delta}\left(\Psi_{r}(\theta)-\Psi_{r}(0)\right) K_{f}(\varrho, \theta) d \theta d \varrho\right| \\
& \quad \leq \operatorname{const}(1-r) \int_{1}^{\infty} \int_{|\theta|<\delta} \frac{1}{\varrho^{2}|\sin (\theta)|} \theta^{2} d \theta \leq \operatorname{const}(1-r) .
\end{aligned}
$$

It is also clear that the second term of the sum (4.4) converges to 0 as $r$ tends to 1 , so (4.3) holds. Replacing $\Psi_{r}(0)$ by $\Psi_{r}(\pi)$ and $-c_{0}+d_{0}$ by $d_{0}$ in the above proof we also obtain the analogue of (4.3) where the integration domain $|\theta|<\delta$ is replaced by $|\theta-\pi|<\delta$.

On the other hand, we also have

$$
\lim _{r \rightarrow 1^{-}} \int_{1}^{\infty} \int_{(\delta, \pi-\delta) \cup(\pi+\delta,-\delta)}\left(\Psi_{r}(\theta)-\left(-c_{0}+d_{0} \widetilde{H}(\theta)\right)\right) K_{f}(\varrho, \theta) d \theta d \varrho=0 .
$$

Indeed, let $A$ and $\widetilde{K}_{f}$ be as in Remark 4.1. Let $K_{f}^{*}$ be an extension of $\widetilde{K}_{f}$ belonging to $\mathcal{V}$. Then (4.6) follows from the facts that $\lim _{r \rightarrow 1^{-}}\left\langle\Psi_{r}, K_{f}^{*}\right\rangle=$ $\left\langle\Psi, K_{f}^{*}\right\rangle$ and that for some positive constant and all $\theta \in(-\delta, \delta) \cup(\pi-\delta, \pi+\delta)$ we have $\left|\Psi_{r}(\theta)-\left(-c_{0}+d_{0} \widetilde{H}(\theta)\right)\right| \leq$ const $(1-r) \delta^{2}$.

Finally, since $\int_{0}^{1} K_{f}(\varrho, \cdot) d \varrho$ belongs to $\mathcal{V}$ and since for $0 \leq r<1$ the function $\Psi_{r}(\theta) K_{f}(\varrho, \theta)$ is integrable on $(0,2 \pi) \times(0,1)$ we have

$$
\begin{aligned}
\lim _{r \rightarrow 1^{-}} \int_{0}^{1}\left\langle\Psi_{r}, K_{f}(\varrho, \cdot)\right\rangle d \varrho & =\lim _{r \rightarrow 1^{-}}\left\langle\Psi_{r}, \int_{0}^{1} K_{f}(\varrho, \cdot) d \varrho\right\rangle \\
& =\int_{0}^{2 \pi}\left(c_{0}+d_{0} \widetilde{H}(\theta)\right) \int_{0}^{1} K_{f}(\varrho, \theta) d \varrho d \theta
\end{aligned}
$$

and the lemma follows for $n$ odd.

The proof for $n$ even is similar with $-c_{0}+d_{0} \widetilde{H}(\theta)$ replaced by $-c_{0}$ in the above argument. 
Proposition 4.3. $\left\langle\Phi_{1,1}, f\right\rangle$ is well defined for $f \in S\left(H_{n}\right)$ and

$$
\begin{aligned}
\left\langle\Phi_{1,1}, f\right\rangle= & 4^{n}(n-1) ! c_{0} \int_{\mathbb{R}^{2}} \frac{1}{\left(\tau^{2}+16 t^{2}\right)^{n / 2}} \\
& \times \operatorname{sgn}(\tau)\left(N f(\tau, t)-\sum_{j=0}^{n-2} \frac{\tau^{j}}{j !} \cdot \frac{\partial^{j} N f}{\partial \tau^{j}}(0, t)\right) d \tau d t
\end{aligned}
$$

where $c_{0}$ is the constant of Proposition 3.3.

Proof. The right member of (4.8) is finite by Lemma 2.3. On the other hand, the expression (2.12) that defines $\left\langle\Phi_{1,1}, f\right\rangle$ agrees, term to term, with (2.16) and so, by Lemma 2.3, also agrees with (2.17). Thus the corollary follows from Proposition 4.2.

So $\left\langle\Phi_{1,2}, f\right\rangle$ is also well defined for $f \in S\left(H_{n}\right)$. Our next step will be to compute $\left\langle\Phi_{1,2}, f\right\rangle$.

For $\varepsilon>0, l \in \mathbb{N}$ and $f \in S\left(H_{n}\right)$ we set

$$
d_{\varepsilon, l, f}=\int_{\mathbb{R}^{2}} e^{-\varepsilon|\lambda|}|\lambda|^{n-2-l} e^{-i \lambda t} \frac{\partial^{l} N f}{\partial \tau^{l}}(0, t) d t d \lambda .
$$

So, (2.13) reads

$$
\left\langle\Phi_{1,2}, f\right\rangle=\lim _{r \rightarrow 1^{-}} \lim _{\varepsilon \rightarrow 0^{+}} \sum_{k \geq 0} \sum_{\substack{l \text { odd } \\ 0 \leq l \leq n-2}} 2^{l+2} \frac{r^{2 k+n}}{2 k+n}\left(a_{k, l}+b_{k, l}\right) d_{\varepsilon, l, f}
$$

with $a_{k, l}$ and $b_{k, l}$ defined by (2.10) and (2.9) respectively.

We will need the following

Lemma 4.4. Let $a_{k, l}$ be defined by (2.10). Then

$$
\begin{aligned}
a_{k, l}= & (-1)^{k} \sum_{j=1}^{l+1} \frac{1}{2^{n-l-j-1}}\left(\begin{array}{c}
n-j-1 \\
l-j+1
\end{array}\right)\left(\begin{array}{c}
j+k-1 \\
k
\end{array}\right) \\
& +\sum_{j=1}^{n-l-1} \frac{1}{2^{n-l-j-1}}\left(\begin{array}{c}
n-j-1 \\
l
\end{array}\right)\left(\begin{array}{c}
j+k-1 \\
k
\end{array}\right) .
\end{aligned}
$$

Proof. From the generating identity (3.2) for the Laguerre polynomials we can write, for $0 \leq r<1$,

$$
\begin{aligned}
\sum_{k=0}^{\infty} r^{k} \frac{1}{l !} \int_{0}^{\infty} L_{k}^{n-1}(\tau) e^{-\tau / 2} \tau^{l} d \tau & =\frac{1}{l !} \cdot \frac{1}{(1-r)^{n}} \int_{0}^{\infty} \tau^{l} e^{-(r+1) \tau /(2(1-r))} d \tau \\
& =\frac{2^{l+1}}{(1-r)^{n-l-1}(1+r)^{l+1}}
\end{aligned}
$$


So $a_{k, l}$ is the coefficient of $r^{k}$ in the power expansion at the origin of (4.11). We decompose (4.11) in simple fractions

$$
\frac{2^{l+1}}{(1-r)^{n-l-1}(1+r)^{l+1}}=\sum_{j=1}^{n-l-1} \frac{A_{j}}{(1-r)^{j}}+\sum_{j=1}^{l+1} \frac{B_{j}}{(1+r)^{j}} .
$$

Thus, by the residue theorem, we have, for a small positive $\varrho>0$,

$$
A_{j}=-\frac{2^{l+1}}{2 \pi i} \int_{|z-1|=\varrho} \frac{1}{(1-z)^{n-l-j}(1+z)^{l+1}} d z .
$$

So

$$
A_{j}=-\frac{2^{l+1}(-1)^{n-l-j}}{(n-l-j-1) !}\left(\frac{d}{d z}\right)_{\mid z=1}^{n-l-j-1}\left((1+z)^{-l-1}\right)=\frac{1}{2^{n-l-j-1}}\left(\begin{array}{c}
n-j-1 \\
l
\end{array}\right) .
$$

Similarly,

$$
\begin{aligned}
B_{j} & =\frac{2^{l+1}}{2 \pi i} \int_{|z+1|=\varrho} \frac{1}{(1-z)^{n-l-1}(1+z)^{l-j+2}} d z \\
& =\frac{2^{l+1}}{(l+1-j) !}\left(\frac{d}{d z}\right)_{\mid z=-1}^{l+1-j}\left((1-z)^{l+1-n}\right)=\frac{1}{2^{n-l-j-1}}\left(\begin{array}{c}
n-j-1 \\
n-l-2
\end{array}\right) .
\end{aligned}
$$

Since, for $m \in \mathbb{N} \cup\{0\},\left(\begin{array}{c}m+k-1 \\ k\end{array}\right)$ is the coefficient of $r^{k}$ in the power expansion at the origin of $(1-r)^{-m}$, the lemma follows.

For $T \in S^{\prime}(\mathbb{R})$, we write $\widehat{T}$ for its Fourier transform and, as before, for $g: \mathbb{R} \rightarrow \mathbb{C}$ we set $g^{\vee}(t)=g(-t)$.

LEMMA 4.5. Assume $0 \leq l \leq n-2$. For $\varepsilon>0$ and $f \in S\left(H_{n}\right)$ let $d_{\varepsilon, l, f}$ be defined by (4.9). Then, for $n-l$ odd,

$$
\lim _{\varepsilon \rightarrow 0^{+}} d_{\varepsilon, l, f}=2(-1)^{(n-l-1) / 2}\left\langle\mathrm{p} \cdot \mathrm{v} \cdot\left(\frac{1}{t}\right), \frac{\partial^{n-2} N f}{\partial \tau^{l} \partial \lambda^{n-l-2}}(0, \cdot)\right\rangle,
$$

and for $n-l$ even,

$$
\lim _{\varepsilon \rightarrow 0^{+}} d_{\varepsilon, l, f}=2(-1)^{(n-l-2) / 2} \frac{\partial^{n-2} N f}{\partial \tau^{l} \partial \lambda^{n-l-2}}(0,0) .
$$

Proof. Let $g(\lambda)=e^{-\varepsilon|\lambda|}|\lambda|^{n-2-l}$. Since

$$
d_{\varepsilon, l, f}=\int_{\mathbb{R}} e^{-\varepsilon|\lambda|}|\lambda|^{n-2-l}\left(\frac{\partial^{l} N f}{\partial \tau^{l}}(0, \cdot)\right)^{\wedge}(\lambda) d \lambda,
$$

a computation of $\widehat{g}$ and the properties of the Fourier transform give the assertion. 
Remark 4.6. For $j \in \mathbb{N}$ and $0 \leq r<1$, let

$$
h_{j}(r)=\sum_{k \geq 0} \frac{r^{2 k+n}}{2 k+n}(-1)^{k}\left(\begin{array}{c}
j+k-1 \\
k
\end{array}\right) .
$$

Then $\lim _{r \rightarrow 1^{-}} h_{j}(r)=\int_{0}^{1} r^{j-1}\left(1+r^{2}\right)^{-j} d r$. Indeed, $h_{j}(0)=0$ and proceeding as at the beginning of Section 3 , we now get $h_{j}^{\prime}(r)=r^{j-1} /\left(1+r^{2}\right)^{j}$.

REMARK 4.7. Note that if $P$ is a nonzero polynomial then

$$
\lim _{r \rightarrow 1^{-}} \sum_{k \geq 0} P(k) \frac{r^{2 k+n}}{2 k+n}= \pm \infty .
$$

Indeed, without loss of generality we can assume that the leading term of $P$ has a positive coefficient. Then, for $k$ large enough, $P(k)$ is greater than a positive constant and the assertion follows.

For $1 \leq j \leq n-1$, we set

$$
c_{j}=\int_{0}^{1} \frac{r^{j-1}}{\left(1+r^{2}\right)^{j}} d r .
$$

Proposition 4.8. For $n$ even we have

$$
\begin{aligned}
\left\langle\Phi_{1,2}, f\right\rangle= & \sum_{\substack{1 \leq l \leq n-2 \\
l \text { odd }}} 2^{2 l+3-n}(-1)^{(n-l+1) / 2} \\
& \times \sum_{j=1}^{l+1} 2^{j} c_{j}\left(\begin{array}{c}
n-j-1 \\
l-j+1
\end{array}\right)\left\langle\text { p.v. }\left(\frac{1}{t}\right), \frac{\partial^{n-2} N f}{\partial \tau^{l} \partial \lambda^{n-l-2}}(0, \cdot)\right\rangle
\end{aligned}
$$

and for $n$ odd we have

$$
\begin{aligned}
\left\langle\Phi_{1,2}, f\right\rangle= & \sum_{\substack{1 \leq l \leq n-2 \\
l \text { odd }}} 2^{2 l+3-n}(-1)^{(n-l+2) / 2} \\
& \times \sum_{j=1}^{l+1} 2^{j} c_{j}\left(\begin{array}{c}
n-j-1 \\
l-j+1
\end{array}\right) \frac{\partial^{n-2} N f}{\partial \tau^{l} \partial \lambda^{n-l-2}}(0,0) .
\end{aligned}
$$

Proof. Let $a_{k, l}$ and $b_{k, l}$ be defined by (2.10) and (2.9) respectively. We write $a_{k, l}=a_{k, l}^{\prime}+a_{k, l}^{\prime \prime}$, with

$$
\begin{aligned}
& a_{k, l}^{\prime}=(-1)^{k} \sum_{j=1}^{l+1} \frac{1}{2^{n-l-j-1}}\left(\begin{array}{c}
n-j-1 \\
l-j+1
\end{array}\right)\left(\begin{array}{c}
j+k-1 \\
k
\end{array}\right), \\
& a_{k, l}^{\prime \prime}=\sum_{j=1}^{n-l-1} \frac{1}{2^{n-l-j-1}}\left(\begin{array}{c}
n-j-1 \\
l
\end{array}\right)\left(\begin{array}{c}
j+k-1 \\
k
\end{array}\right) .
\end{aligned}
$$


For $\varepsilon>0,0 \leq l \leq n-2$ and $f \in S\left(H_{n}\right)$ let $d_{\varepsilon, l, f}$ be defined by (4.9). We set

$$
\left\langle\Phi_{1,2}^{*}, f\right\rangle=\lim _{r \rightarrow 1^{-}} \lim _{\varepsilon \rightarrow 0^{+}} \sum_{k \geq 0} \sum_{\substack{0 \leq l \leq n-2 \\ l \text { odd }}} \frac{r^{2 k+n}}{2 k+n} 2^{l+2} d_{\varepsilon, l, f} a_{k, l}^{\prime} .
$$

Note that, by Lemma 4.5 and Remark $4.6,\left\langle\Phi_{1,2}^{*}, f\right\rangle$ is well defined, and moreover

$$
\left\langle\Phi_{1,2}^{*}, f\right\rangle=\sum_{\substack{0 \leq l \leq n-2 \\
l \text { odd }}} 2^{l+2} \lim _{\varepsilon \rightarrow 0^{+}} d_{\varepsilon, l, f} \sum_{j=1}^{l+1} \frac{1}{2^{n-l-j-1}}\left(\begin{array}{c}
n-j-1 \\
l-j+1
\end{array}\right) c_{j} .
$$

So, the functional $\Phi_{1,2}^{* *}$ defined by

$$
\left\langle\Phi_{1,2}^{* *}, f\right\rangle=\lim _{r \rightarrow 1^{-}} \lim _{\varepsilon \rightarrow 0^{+}} \sum_{k \geq 0} \sum_{\substack{0 \leq l \leq n-2 \\ l \text { odd }}} 2^{l+2} \frac{r^{2 k+n}}{2 k+n} d_{\varepsilon, l, f}\left(a_{k, l}^{\prime \prime}+b_{k, l}\right)
$$

is also well defined. Now, given $\varphi \in S(\mathbb{R})$, it is easy to see that there exists $f \in S\left(H_{n}\right)$ such that $\partial^{m} N f(0, t) / \partial \tau^{m}=\delta_{l, m} \varphi(t)$ for $m=0, \ldots, n-2, t \in \mathbb{R}$. Indeed, pick $g \in S(\mathbb{R})$ such that $g^{(j)}(0)=\delta_{j, l}$ for $j=0,1, \ldots, n-2$. Since $N: S\left(\mathbb{C}^{n}\right) \rightarrow \mathcal{H}$ is surjective, there exists $\widetilde{g} \in S\left(\mathbb{C}^{n}\right)$ such that $N \widetilde{g}=g$. Now, $f(z, t)=\widetilde{g}(z) \varphi(t)$ has the desired property. So for each $l$ odd with $0 \leq l \leq n-2$ the limit

$$
\lim _{r \rightarrow 1^{-}} \sum_{k \geq 0} \frac{r^{2 k+n}}{2 k+n}\left(a_{k, l}^{\prime \prime}+b_{k, l}\right)
$$

exists and is finite. But $a_{k, l}^{\prime \prime}+b_{k, l}$ is a polynomial in $k$, so Remark 4.7 implies that $a_{k, l}^{\prime \prime}+b_{k, l}$ vanishes identically at $k$ and so $\left\langle\Phi_{1,2}^{* *}, f\right\rangle=0$. Now, (4.15) and Lemma 4.5 give the assertion.

Remark 4.9. Let $\Phi_{2}$ be as in (2.5). Then

$$
\begin{aligned}
\left\langle\Phi_{2}, f\right\rangle= & \sum_{l=0}^{n-2} \sum_{\substack{1 \leq k \leq n-1 \\
k \neq n / 2}} \frac{1}{n-2 k} \\
& \times \sum_{\max (n-k-1, l) \leq j \leq n-2}\left(\begin{array}{l}
j \\
l
\end{array}\right)\left(\begin{array}{c}
k-1 \\
n-j-2
\end{array}\right) 2^{2 l+3-n-j}\left\langle T_{l}, f\right\rangle
\end{aligned}
$$

where

$$
\left\langle T_{l}, f\right\rangle=(-1)^{(n-l-1+2 j) / 2}\left\langle\text { p.v. }\left(\frac{1}{t}\right), \frac{\partial^{n-2} N f}{\partial \tau^{l} \partial t^{n-l-2}}(0, \cdot)\right\rangle
$$


if $n-l$ is odd, and

$$
\left\langle T_{l}, f\right\rangle=(-1)^{(n-l+2 j) / 2} \frac{\partial^{n-2} N f}{\partial \tau^{l} \partial t^{n-l-2}}(0,0)
$$

if $n-l$ is even.

Indeed, to see (4.16), we recall that $S_{\lambda, k}=F_{\lambda, k} \otimes e^{-i \lambda t}$. Then a change of indices in (2.3) gives

$$
\begin{aligned}
\left\langle\Phi_{2}, f\right\rangle= & \sum_{l=0}^{n-2} \sum_{\substack{1 \leq k \leq n-1 \\
k \neq n / 2}} \frac{1}{n-2 k} \\
& \times \sum_{\max (n-k-1, l) \leq j \leq n-2}(-1)^{n-j+l}\left(\begin{array}{l}
j \\
l
\end{array}\right)\left(\begin{array}{c}
k-1 \\
n-j-2
\end{array}\right) 2^{2 l+2-n-j} d_{\varepsilon, l, f}
\end{aligned}
$$

so the assertion follows from Lemma 4.5.

Now, we can state

THEOREM 4.10. There exist constants $c_{0}, e_{0}, \ldots, e_{n-2}, e_{0}^{\prime}, \ldots, e_{n-2}^{\prime}$ such that, for $f \in S\left(H_{n}\right)$,

$$
\begin{aligned}
\langle\Phi, f\rangle= & 4^{n}(n-1) ! c_{0} \int \frac{\operatorname{sgn}(\tau)}{\left(\tau^{2}+16 t^{2}\right)^{n / 2}}\left(N f(\tau, t)-\sum_{l=0}^{n-2} \frac{\tau^{l}}{l !} \cdot \frac{\partial^{l} N f}{\partial \tau^{l}}(0, t)\right) d \tau d t \\
& +\sum_{l=0}^{n-2} e_{l}\left\langle\text { p.v. }\left(\frac{1}{t}\right), \frac{\partial^{n-2} N f}{\partial \tau^{l} \partial t^{n-2-l}}(0, \cdot)\right\rangle+\sum_{l=0}^{n-2} e_{l}^{\prime} \frac{\partial^{n-2} N f}{\partial \tau^{l} \partial t^{n-2-l}}(0,0) .
\end{aligned}
$$

Proof. Since $\Phi=\Phi_{1,1}+\Phi_{1,2}+\Phi_{2}$, the theorem follows from Propositions $4.3,4.8$ and 4.9 .

Finally, we remark that the constants $c_{0}, e_{0}, \ldots, e_{n-2}, e_{0}^{\prime}, \ldots, e_{n-2}^{\prime}$ in the above theorem can be explicitly computed; in fact, the value of $c_{0}$ is found from Proposition 4.3 and (3.4) and the values of $e_{0}, \ldots, e_{n-2}, e_{0}^{\prime}, \ldots, e_{n-2}^{\prime}$ are found from (4.13), (4.14) and (4.16).

\section{References}

[B-D-R] C. Benson, A. H. Dooley and G. Ratcliff, Fundamental solutions for powers of the Heisenberg sub-Laplacian, Illinois J. Math. 37 (1993), 455-476.

[F1] G. A. Folland, A fundamental solution for a subelliptic operator, Bull. Amer. Math. Soc. 79 (1973), 373-376.

[F2] - Hermite distributions associated to the group $U(p, q)$, Proc. Amer. Math. Soc. 126 (1998), 1751-1763.

[G-S] T. Godoy and L. Saal, $L^{2}$ spectral decomposition on the Heisenberg group associated to the action of $U(p, q)$, Pacific J. Math. 193 (2000), 327-353. 
[H-T] R. Howe and E. C. Tan, Nonabelian Harmonic Analysis, Springer, New York, 1992.

[M-R1] D. Müller and F. Ricci, Analysis of second order differential operators on Heisenberg groups I, Invent. Math. 101 (1990), 545-582.

[M-R2] - - - Analysis of second order differential operators on Heisenberg groups II, J. Funct. Anal. 108 (1992), 296-346.

[Sz] G. Szegő, Orthogonal Polynomials, Colloq. Publ. 23, Amer. Math. Soc., 1939.

[T] A. Tengstrand, Distributions invariant under an orthogonal group of arbitrary signature, Math. Scand. 8 (1960), 201-218.

Facultad de Matematica, Astronomia y Fisica

Universidad Nacional de Cordoba

Ciudad Universitaria

5000 Cordoba, Argentina

E-mail: godoy@mate.uncor.edu

Received March 27, 2000

Revised version September 28, 2000 